\title{
Heart Rate Recovery Is Impaired in Patients with Inflammatory Bowel Diseases
}

\author{
Bahadir Sarli Yasemin Dogan Orhan Poyrazoglu Ahmet Oguz Baktir \\ Abdullah Eyvaz Engin Altinkaya Ahmet Tok Engin Donudurmaci \\ Mehmet Ugurlu Adile Ortakoyluoglu Hayrettin Saglam Huseyin Arinc
}

Departments of Cardiology and Gastroenterology, Kayseri Education and Research Hospital, Kayseri, Turkey

\section{Key Words}

Inflammatory bowel disease $\cdot$ Heart rate recovery index .

Cardiovascular risk

\begin{abstract}
Objective: In this study we aimed to investigate heart rate recovery (HRR) in patients with inflammatory bowel disease (IBD). Subjects and Methods: A total of 40 patients with IBD and 30 healthy controls were included in this study. A treadmill stress test was performed in all the patients to calculate the HRR index based on the age-specific maximum heart rate. The HRR indices were calculated as follows: $\mathrm{HRR}_{1,2,3,4,5}=$ heart rate at peak exercise - heart rate at 1, 2, 3,4 , and $5 \mathrm{~min}$. The independent samples $t$ test was used to compare HRR indices between the patient and control groups. The Pearson correlation coefficient was used to examine the association between the duration of IBD and the HRR indices. Multivariate regression analysis was carried out to identify predictors of impaired HRR in patients with IBD. Results: HRR indices at various time intervals were significantly lower in the patients with IBD than in the controls: $\mathrm{HRR}_{1}(1.18 \pm 8$ vs. $31 \pm 7, \mathrm{p}<0.001), \mathrm{HRR}_{2}(36 \pm 12$ vs. $51 \pm 8$, $\mathrm{p}<0.001), \mathrm{HRR}_{3}(46 \pm 12$ vs. $62 \pm 11, \mathrm{p}<0.001), \mathrm{HRR}_{4}(54 \pm 7$
\end{abstract}

\begin{tabular}{ll}
\hline KARGER & $\begin{array}{l}\text { ( } 2016 \text { S. Karger AG, Basel } \\
1011-7571 / 16 / 0254-0363 \$ 39.50 / 0\end{array}$ \\
$\begin{array}{l}\text { E-Mail karger@karger.com } \\
\text { www.karger.com/mpp }\end{array}$ & $\begin{array}{l}\text { This is an Open Access article licensed under the terms of the } \\
\text { Creative Commons Attribution-NonCommercial 3.0 Un- } \\
\text { ported license (CC BY-NC) (www.karger.com/OA-license), } \\
\text { applicable to the online version of the article only. Distribu- } \\
\text { tion permitted for non-commercial purposes only. }\end{array}$
\end{tabular}

vs. $65 \pm 8, \mathrm{p}<0.001)$, and $\mathrm{HRR}_{5}(55 \pm 13$ vs. $71 \pm 15, \mathrm{p}<0.001)$. Mean duration of IBD was $7.8 \pm 3.6$ years. In addition, there was a significant negative correlation between disease duration and HRR at the first minute $(r=-0.704, p<0.001)$. Multivariate logistic regression analysis showed that symptom duration (OR: $1.742,95 \% \mathrm{Cl}: 1.148-2.636, \mathrm{p}=0.009$ ) was an independent predictor of impaired HRR in patients with IBD. Conclusion: In this study, the data showed that the HRR was impaired in patients with IBDs. Hence, given the prognostic value of the test, patients with IBD should be monitored for future cardiovascular events.

(c) 2016 S. Karger AG, Basel

\section{Introduction}

Inflammatory bowel diseases (IBDs) are chronic inflammatory disorders that can involve several regions and layers of the gastrointestinal tract and can be characterized by remissions and exacerbations [1]. Although the etiology of IBDs has not been fully elucidated, genetic and environmental factors, host immune response, and autonomic nervous system dysfunction have been implicated [2]. 
Fig. 1. HRR indices of patients with IBDs and controls at 1, 2, 3 and 5 min of recovery.

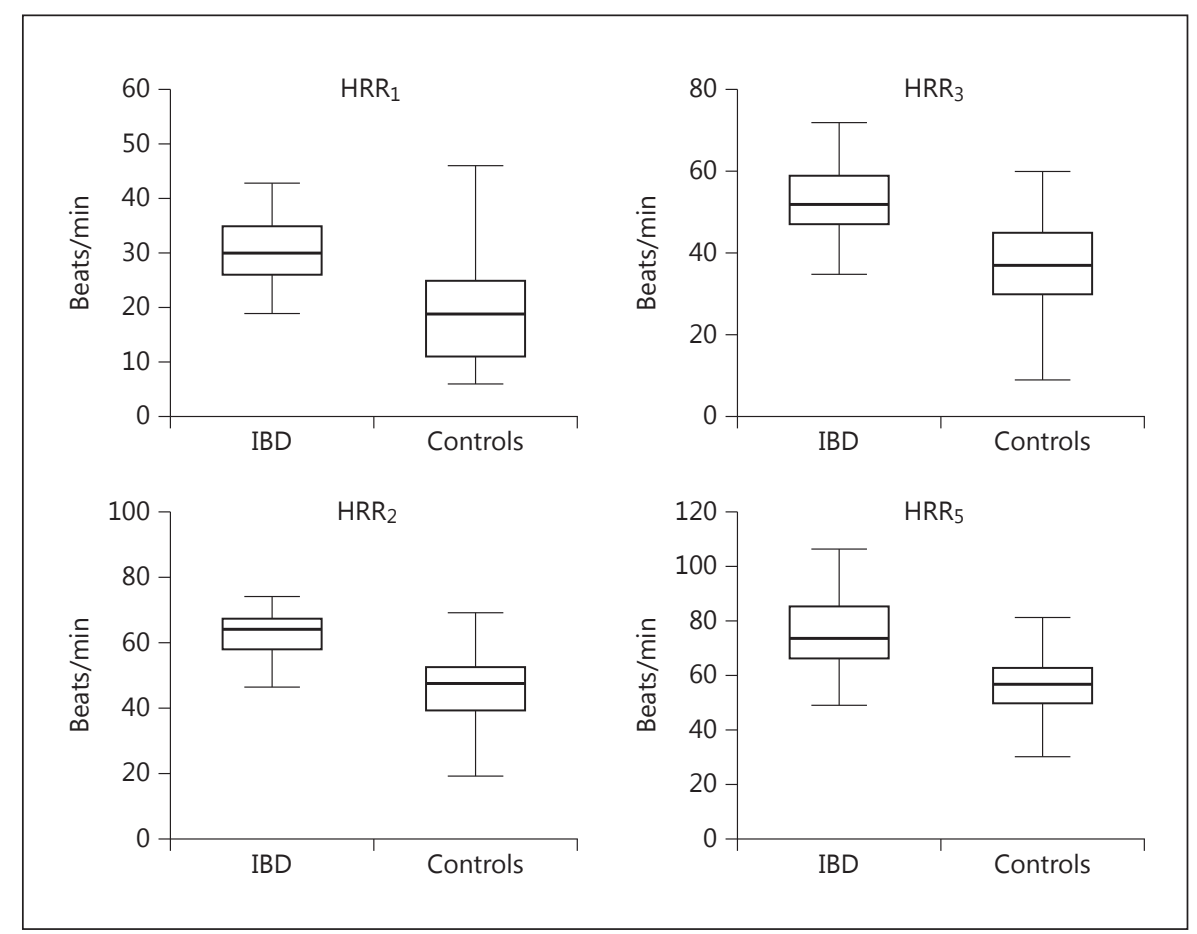

There have been several reports [3,4] of the risk of cardiovascular disease in IBD, a chronic inflammatory disease of the gut. It has been shown that IBD is associated with an increased risk of cardiovascular death, particularly in the active period of the disease [4]. There is an increasing body of evidence suggesting a complex interplay between inflammatory cells, the immune system, and the enteric autonomic nervous system [5]. It was thought that autonomic nerve system dysfunction might have a role in pathogenesis of IBD [6]. An abnormal hypothalamic-pituitary-adrenal axis and cholinergic antiinflammatory pathway, a deleterious effect of stress and depression as well as an abnormal coupling of the prefrontal cortex-amygdala complex could play a role in the pathogenesis of IBD [7].

Heart rate recovery (HRR) is defined as the decrease in heart rate after exercise. HRR is estimated by obtaining heart rates at minutes $1,2,3$, and 5 during the recovery period at maximal heart rate in a patient undergoing a maximal stress test. Impaired HRR had been reported as an independent predictor of mortality in both men and women [8]. A correlation had been shown between the amount of reduction in heart rate at the first minute of recovery and mortality, where mortality decreases as the amount of reduction increases [9]. Therefore, in the present study, we aimed to investigate the HRR indices in patients with IBD.

\section{Subjects and Methods}

\section{Patient Selection}

A total of 44 consecutive patients who had a diagnosis of IBD at the Gastroenterology Outpatient Clinic, Kayseri Education and Research Hospital between December 2014 and June 2015 were included in this cross-sectional study. A group of 30 healthy controls attending the Cardiology Outpatient Clinic and free from cardiovascular diseases were selected as the control group. Exclusion criteria were age $<15$ and $>70$ years, a history of previous myocardial infarction, ischemic heart disease, left ventricular ejection fraction $<50 \%$, severe valvular failure or stenosis, cardiomyopathy, cardiac arrhythmia including atrial fibrillation, comorbid diseases such as hypertension, diabetes mellitus, chronic renal failure, $\mathrm{Hb}<10 \mathrm{~g} / \mathrm{dl}$, autonomic system disorder, and rheumatoid diseases. Based on the exclusion criteria, 4 patients were not included in the study: 2 had renal failure, 1 had a previous myocardial infarction, and another one had diabetes). Thus, 40 patients (ulcerative colitis: 22 and Crohn's disease: 18) with IBD and 30 healthy controls were enrolled.

Patients' total cholesterol, high-density lipoprotein cholesterol, low-density lipoprotein cholesterol, fasting glucose, and creatinine levels were retrieved from medical records. The study was approved by the Institutional Ethics Committee. All enrolled patients had given written informed consent.

\section{Stress Test}

Baseline ECG was evaluated in all patients with IBD and in healthy controls. In addition, all participants underwent transthoracic echocardiography. A stress test was performed to calculate the HRR index based on age-specific maximum heart rate.

The stress test was performed according to the Bruce protocol. Maximum heart rate was calculated as follows: maximum heart 
rate $=220$ - age. Patients who achieved $85 \%$ of maximum heart rate were included in the study. The ECG was recorded continuously during the exercise test. At the end of the exercise test, the heart rate was recorded for 5 min during the period of time after exercise with the subject at rest in the supine position. HRR indices were calculated by subtracting heart rates at the 1 st $\left(\mathrm{HRR}_{1}\right), 2 \mathrm{nd}$ $\left(\mathrm{HRR}_{2}\right), 3 \mathrm{rd}\left(\mathrm{HRR}_{3}\right), 4$ th $\left(\mathrm{HRR}_{4}\right)$, and 5 th $\left(\mathrm{HRR}_{5}\right)$ minutes during the recovery period from the heart rate at peak exercise. HRR indices were calculated as follows: $\mathrm{HRR}_{1,2,3,4,5}=$ heart rate at peak exercise - heart rate at $1,2,3,4$, and $5 \mathrm{~min}$.

\section{Statistical Analysis}

All analyses were carried out using SPSS 21.0 for Windows (SPSS Inc., Armonk, N.Y., USA). Continuous variables were given as mean \pm standard deviation; categorical variables were defined as percentages. The variables were investigated using the Kolmogorov-Smirnov test to determine whether or not they are normally distributed. The independent samples test was used to compare continuous variables between the two groups. The $\chi^{2}$ test was used to compare categorical data. The Pearson correlation coefficient was used to examine the association between the duration of IBD and the HRR indices. Multivariate logistic regression analyses were done to identify predictors of impaired $H_{R} R_{1}$ in patients with IBD. A two-tailed $\mathrm{p}$ value $<0.05$ was considered as significant.

\section{Results}

The baseline characteristics of the study population are shown in table 1 . The IBD and control groups were similar with respect to age, BMI, total cholesterol, highdensity lipoprotein cholesterol, low-density lipoprotein cholesterol, and fasting glucose, potassium and creatinine levels. There were no significant differences in the left ventricular ejection fraction between patients and controls $(64.1 \pm 2$ to $64.6 \pm 3 \%, \mathrm{p}=0.357)$. In patients with IBD, the mean duration of the disease was $7.8 \pm 3.6$ years.

All patients included in the study achieved the target heart rate at the exercise test. Comparison of the maximal heart rate, maximal systolic and diastolic blood pressure, exercise duration, and metabolic equivalents (METs) achieved during the exercise stress test between patients with IBD and controls are summarized in table 2. Patients with IBD and controls were similar in terms of duration of exercise $(12.1 \pm 1$ to $11.9 \pm 1 \mathrm{~min}, \mathrm{p}=0.621)$, METs (12.5 \pm 1 to $12.2 \pm 1$ METs, $\mathrm{p}=0.245)$, and maximal heart rate ( $164 \pm 10$ to $168 \pm 13, \mathrm{p}=0.179)$ achieved during the exercise stress test.

The HRR indices of the IBD group and the control group are shown in table 2. The HRR indices were significantly lower in the IBD group compared to the healthy controls (fig. 1). Of 40 patients with IBD, 18
Table 1. Demographic and clinical features of the patients with IBD and controls

\begin{tabular}{lccc}
\hline & $\begin{array}{l}\text { IBD } \\
\text { group } \\
(\mathrm{n}=40)\end{array}$ & $\begin{array}{l}\text { Control } \\
\text { group } \\
(\mathrm{n}=30)\end{array}$ & p value \\
\hline Age, years & $36.6 \pm 6$ & $35.9 \pm 6$ & 0.642 \\
Gender (M/F) & $24 / 16$ & $17 / 13$ & 0.779 \\
BMI & $23.9 \pm 3$ & $23.7 \pm 3$ & 0.744 \\
Ejection fraction, \% & $64.1 \pm 2$ & $64.6 \pm 3$ & 0.357 \\
Fasting glucose, mg/dl & $93 \pm 12$ & $94 \pm 8$ & 0.636 \\
Potassium level, mmol/l & 4.1 & 4.2 & 0.211 \\
Triglycerides, mg/dl & $120 \pm 51$ & $131 \pm 73$ & 0.432 \\
LDL cholesterol, mg/dl & $88 \pm 28$ & $98 \pm 39$ & 0.242 \\
HDL cholesterol, mg/dl & $42 \pm 8$ & $40 \pm 6$ & 0.369 \\
Creatinine, mg/dl & $0.8 \pm 0.2$ & $0.8 \pm 0.1$ & 0.641 \\
Duration of IBD, years & $7.8 \pm 3.6$ & & \\
\hline
\end{tabular}

$\mathrm{HDL}=$ High-density lipoprotein LDL = low-density lipoprotein.

Table 2. Comparison of exercise test results between the patients with IBD and the controls

\begin{tabular}{lccr}
\hline & $\begin{array}{l}\text { IBD } \\
\text { group } \\
(\mathrm{n}=40)\end{array}$ & $\begin{array}{l}\text { Control } \\
\text { group } \\
(\mathrm{n}=30)\end{array}$ & p value \\
\hline Duration of exercise, min & $12.1 \pm 1$ & $11.9 \pm 1$ & 0.621 \\
Maximum heart rate, beats/min & $164 \pm 10$ & $168 \pm 13$ & 0.179 \\
Maximum systolic BP, mm Hg & $171 \pm 14$ & $167 \pm 17$ & 0.336 \\
Maximum diastolic BP, mm Hg & $90 \pm 11$ & $88 \pm 18$ & 0.491 \\
Maximal MET & $12.5 \pm 1$ & $12.2 \pm 1$ & 0.245 \\
$\mathrm{HRR}_{1}$ & $18 \pm 8$ & $31 \pm 7$ & $<0.001$ \\
$\mathrm{HRR}_{2}$ & $36 \pm 12$ & $51 \pm 8$ & $<0.001$ \\
$\mathrm{HRR}_{3}$ & $46 \pm 12$ & $62 \pm 11$ & $<0.001$ \\
$\mathrm{HRR}_{5}$ & $55 \pm 13$ & $71 \pm 15$ & $<0.001$ \\
\hline
\end{tabular}

$\mathrm{BP}=$ Blood pressure

(45\%) had an $\mathrm{HRR}_{1}$ under 12 beats/min. There was a significant negative correlation between the duration of symptoms and $\operatorname{HRR}_{1}(\mathrm{r}=-0.704, \mathrm{p}<0.001)$. Multivariate logistic regression analysis showed that in the patients with IBD, symptom duration (OR: 1.742, 95\% CI: $1.148-2.636, \mathrm{p}=0.009)$ was an independent predictor of impaired $\mathrm{HRR}_{1}(<12 / \mathrm{min})$ among variables that included age, male gender, presence of ulcerative colitis, potassium level, and left ventricular ejection fraction in patients with IBD. 


\section{Discussion}

In this study, the HRR indices were significantly lower in the patients with IBD than in the healthy controls. In addition, there was a significant negative correlation between the duration of IBD and the HRR indices. The relation between IBD and HRR has not been studied before. Our study is the first one which shows the lower HRR indices in patients with IBD.

Crohn's disease and ulcerative colitis are chronic inflammatory diseases of the bowel. Some reports had shown that the risk of atrial fibrillation, myocardial infarction, and cardiovascular death is increased in these patients particularly in the active period of the disease [10]. However, the pathophysiological mechanisms leading to increased cardiovascular death in this patient population are not clear yet. Increased atherothrombosis and venous thromboembolic disease are the suspected clinical precursors of the increased cardiovascular death.

HRR is a powerful measure of autonomic nervous system function and parasympathetic activity. The autonomic nervous system is the main modulator of the heart rate and HRR after exercise. At the end of exercise, parasympathetic reactivation plays a dominant role in modulating heart rate especially at the first minute after exercise. Pierpont et al. [11] showed that HRR during the 1st minute of recovery was independently associated with cardiovascular death and all-cause mortality. Many studies showed that HRR was impaired in patients with chronic inflammatory diseases. Dogdu et al. [12] showed that the HRR index had deteriorated in patients with systemic lupus erythematosus compared to healthy controls. The authors concluded that the multisystemic inflammatory damage existing in patients with systemic lupus erythematosus might lead to malfunction of the autonomic nervous system. In another study, Ardic et al. [13] showed that the HRR index was impaired in patients with sarcoidosis compared to healthy controls. In addition, impaired HRR indices were also reported in patients with Behçet's disease and psoriasis $[14,15]$. The chronic inflammatory process is the common point of all these chronic diseases. Vagal tonus is crucial for the proper functioning of the gastrointestinal system. A decline in vagal tonus might lead to a dysfunction of the gastrointestinal tract [16]. Data demonstrating the simultaneous appearance of both autonomic dysfunction and IBD corroborate the suggestions that autonomic dysfunction plays a role in the development of IBD. Jelenova et al. [17] had previously shown that heart rate variability which is a marker of autonomic dysfunction was blunted in ado- lescents with IBD suggestive of autonomic nervous system dysfunction in these patient populations. A lower vagal tonus was observed in patients with in functional digestive disorders and IBDs. In a previous study, Sharma et al. [18] had shown that patients with IBD, particularly those with ulcerative colitis, had significantly lower parasympathetic activity. Similarly, Ganguli et al. [19] reported that sympathetic activity was increased in patients with ulcerative colitis independent of symptom severity.

Similar to previous studies, our data show that the HRR index was impaired in patients with IBD. We also showed that impairment in HRR indices was correlated with the duration of the IBD. Different from previous studies, we used the HRR index to investigate the autonomic function instead of heart rate variability. HRR is a unique indicator of autonomic nervous function and is well correlated with parasympathetic activity. In addition, HRR may independently predict cardiovascular and all-cause mortality. Besides atherothrombosis and venous thromboembolic disease, another cause of increased cardiovascular mortality observed in IBD patients might be the deteriorated autonomic dysfunction which has been shown by impaired HRR indices. As previously shown in patients with rheumatoid arthritis, chronic inflammation in patients with chronic IBDs might have a role in the deterioration of the autonomic function [20].

This study has several limitations: it is a single-center, cross-sectional, nonrandomized trial, it has a small sample size, the controls were selected from individuals attending the Cardiology Outpatient Clinic, the physical conditioning of the patients and controls was not evaluated, and the medical history including previous $\beta$-blocker treatment and specific treatment targeting IBD was not recorded in detail.

\section{Conclusion}

In this study, HRR, an indicator of autonomic dysfunction, was impaired in patients with IBDs. Given the prognostic value of the test, HRR indices might be beneficial to establish the high-risk subsets for cardiovascular events.

\section{Disclosure Statement}

The authors have no conflicts of interest to declare. 


\section{References}

1 Scott MM, Ekbom A: Epidemiology of inflammatory bowel disease. Curr Opin Gastroenterol 2002;18:416-420.

$\checkmark 2$ Dorofeev AÉ, Shvets OV: Epidemiology and risk factors of inflammatory bowel diseases. Lik Sprava 2014;11:22-29.

-3 Rungoe C, Nyboe Andersen N, Jess T: Inflammatory bowel disease and risk of coronary heart disease. Trends Cardiovasc Med 2015; 25:699-704.

-4 Kristensen SL, Ahlehoff O, Lindhardsen J, et al: Disease activity in inflammatory bowel disease is associated with increased risk of myocardial infarction, stroke and cardiovascular death - a Danish nationwide cohort study. PLoS One 2013;8:e56944.

-5 Buckley MM, O'Mahony SM, O’Malley D, et al: Convergence of neuro-endocrine-immune pathways in the pathophysiology of irritable bowel syndrome. World J Gastroenterol 2014; 20:8846-8858.

6 Bonaz BL, Bernstein CN: Brain-gut interactions in inflammatory bowel disease. Gastroenterology 2013;144:36-49.

7 Bonaz B: Inflammatory bowel diseases: a dysfunction of brain-gut interactions? Minerva Gastroenterol Dietol 2013;59:241-259.
8 Cole CR, Blackstone EH, Pashkow FJ, et al: Heart-rate recovery immediately after exercise as a predictor of mortality. N Engl J Med 1999;341:1351-1357.

-9 Jouven X, Empana JP, Schwartz PJ, et al: Heart-rate profile during exercise as a predictor of sudden death. N Engl J Med 2005;352: 1951-1958.

10 Kristensen SL, Lindhardsen J, Ahlehoff O, et al: Increased risk of atrial fibrillation and stroke during active stages of inflammatory bowel disease: a nationwide study. Europace 2014;16:477-484.

11 Pierpont GL, Stolpman DR, Gornick CC: Heart rate recovery post-exercise as an index of parasympathetic activity. J Auton Nerv Syst 2000;80:169-174.

12 Dogdu O, Yarlioglues M, Kaya MG, et al: Deterioration of heart rate recovery index in patients with systemic lupus erythematosus. J Rheumatol 2010;37:2511-2515.

13 Ardic I, Kaya MG, Yarlioglues M, et al: Impaired heart rate recovery index in patients with sarcoidosis. Chest 2011;139:60-68.
14 Kaya EB, Yorgun H, Akdogan A, et al: Heartrate recovery index is impaired in Behçet's disease. Tex Heart Inst J 2009;36:282-286.

15 Sarli B, Dogan Y, Baktir AO, et al: Heart rate recovery is impaired in patients with psoriasis. Med Princ Pract 2013;22:567-570.

16 Bonaz B, Sinniger V, Pellissier S: Vagal tone: effects on sensitivity, motility, and inflammation. Neurogastroenterol Motil 2016;28:455462.

17 Jelenova D, Ociskova M, Prasko J, et al: Heart rate variability in children with inflammatory bowel diseases. Neuro Endocrinol Lett 2015; 36:72-79.

18 Sharma P, Makharia GK, Ahuja V, et al: Autonomic dysfunctions in patients with inflammatory bowel disease in clinical remission. Dig Dis Sci 2009;54:853-861.

19 Ganguli SC, Kamath MV, Redmond K, et al: A comparison of autonomic function in patients with inflammatory bowel disease and in healthy controls. Neurogastroenterol Motil 2007;19:961-967.

20 Adlan AM, Lip GY, Paton JF, et al: Autonomic function and rheumatoid arthritis: a systematic review. Semin Arthritis Rheum 2014; 44:283-304. 